\title{
Participatory action research
}

David F Marks

Potential competing interests: The author(s) declared that no potential competing interests exist.

Participatory action research is a version of action research that deliberately seeks to provoke some form of social or community change. 